\title{
Prise en charge communautaire des cas de morsure de serpent dans l'aire du centre de santé communautaire et universitaire (CSCom U) de Ségué, Mali
}

\author{
Community management of snakebite cases \\ in the area of the community and university health center (CSCom U) in Segue, Mali
}

\author{
Konaté $\mathrm{A}^{1 *}$, Tembiné $\mathrm{I}^{1}$, Bengaly $\mathrm{I}^{2}$, Coulibaly $\mathrm{MB}^{3}$, Coulibaly $\mathrm{KB}^{4}$, Dakouo $\mathrm{F}^{5}$, Kamissoko $\mathrm{CO}^{6}$, Coulibaly $\mathrm{E}^{7}$, Dicko $\mathrm{FT}^{8,9}$.
}

\section{DOI : 10.53318/msp.v11i1.1890}

1 : Centre de Santé Communautaire et Universitaire de Ségue (Koulikoro-Mali)

2 : Centre de Santé Communautaire et Universitaire de Koniakary (Kayes-Mali)

3: Centre de Santé Communautaire et Universitaire de Konobougou (Ségou-Mali)

4 : Centre de Santé Communautaire et Universitaire de Sanoubougou 2 (Sikasso-Mali)

5 : Centre de santé de référence de Bankass (Mopti-Mali)

6 : Centre de santé de référence de Sélingué (Sikasso-Mali)

7 : Centre de santé de référence de Kolokani (Koulikoro-Mali)

8 : Service de pédiatrie au CHU de Gabriel Touré (Bamako- Mali)

9 : Département de médecine de famille/médecine communautaire ;

FMOS (Bamako-Mali)

Correspondance : Dr Aboubakary KONATE, Centre de Santé Communautaire Universitaire (CSCom U) de Ségué, Bamako -Mali : Tel : (00223) 66562052 ; Email : aboubakarykonate@gmail.com

\section{Résumé}

Introduction : La morsure de serpent est un problème de santé publique et fait partie des pathologies négligées selon l'Organisation Mondiale de la Santé (OMS). L'objectif était d'évaluer la prise en charge des morsures de serpent en milieu rural. Matériel et méthodes : ॥ s'agissait d'une étude prospective qui s'est déroulée du 1er Janvier au 31 Décembre 2020 dans l'aire du centre de santé communautaire et universitaire (CSCom-U) de Ségué. Résultats : Nous avons enregistré dans l'aire de santé de Ségué 25 cas de morsure dont 10 cas (40\%) ont été pris en charge au centre de santé et $15(60 \%)$ dans la communauté. La tranche d'âge 15 à 30 ans était la plus touchée (40\%), le sexe masculin était de $64 \%$ et les cultivateurs étaient les plus touchés avec $36 \%$ des cas. Les facteurs à risque pour la morsure étaient la brousse $(76 \%)$, la saison des pluies $(64 \%)$ et les travaux champêtres (40\%). Nous avons enregistré $100 \%$ de guérison dont un seul cas avec séquelle qui avait été traité traditionnellement. Conclusion : II ressort que la morsure de serpents est un évènement assez négligé dans nos programmes de santé, très souvent les victimes font recours aux soins traditionnels, or l'utilisation du sérum antivenimeux est le traitement de choix. La disponibilité au niveau local des sérums anti venimeux (SAV), la formation continue des agents de santé, et l'éducation de la population doivent être de mise pour améliorer la prise en charge communautaire des envenimations par morsure de serpent.

Mots clés : Prise en charge, morsure, serpent, CSCom U Ségué, Mali

\section{Abstract}

Introduction: The snake bite is a public health problem, one of the neglected pathologies according to the WHO. The objective was to assess the management of snake bites in rural areas. Material and methods: This was a prospective study that took place over a 12-month period from January 1 to December 31, 2020 in the area of the community and university health center (CSCom-U) of Segue. Results: We recorded 25 cases of bite, including 10 cases $(40 \%)$ in the center and $15(60 \%)$ in the community. The $15-30$ age group was the most affected $(40 \%)$, the male was $64 \%$; growers were the most affected with $36 \%$ of cases. The bush, the rainy season and the field work were risk factors for the bite with respectively $76 \%, 64 \%$ and $40 \%$. We recorded $100 \%$ recovery, including only one case with sequelae that had been treated traditionally. Conclusion: Snake bite is a quite neglected event in our health programs, very often victims resort to traditional care, and the use of antivenom is the treatment of choice. The availability at the local level of anti-venomous, the continuous training of health workers, and the education of the population must be in order to improve community management of snakebite envenomation.

Keywords: Management, snake bite, CSCom U Segue, Mali

\section{Introduction :}

L'envenimation par morsure de serpent (EMS) est la conséquence de la rencontre accidentelle d'un homme avec un serpent. Les activités économiques et les occupations du premier, l'écologie et les comportements du second permettent d'expliquer cette rencontre [1]. Déclarées comme "maladie négligée" par l'OMS, les morsures de serpents sont responsables de 421000 cas d'envenimations et de 20000 décès annuellement dans le monde [2]. Les vipéridés et les élapidés sont les deux familles de serpents venimeux, les plus importantes dans le monde, responsables d'envenimations [3]. Les trois quarts des morsures surviennent au cours des travaux agricoles, de la chasse ou du déplacement pédestres en rapport avec le travail [4]. Les hommes subissent entre 50 et $75 \%$ des morsures. Les femmes et les enfants sont moins souvent mordus [5]. La majorité des morsures se produisent en fin d'après-midi ou en début de soirée, quelques-unes ont lieu la nuit à domicile et sont infligées au cours du sommeil par des serpents circulant dans la maison en quête de nourriture [5]. Les villes ne sont pas épargnées par ce phénomène ; même si l'incidence des morsures y est dix à vingt fois plus 
faible qu'en zone rurale [6]. La gravité des envenimations est difficile à apprécier en raison de l'absence de statistiques fiables [7] ; mais aussi du fait que les conditions ne sont actuellement pas réunies pour faciliter la prise en charge des envenimations. A cela s'ajoute le coût dissuasif du sérum antivenimeux, dont la conservation est souvent difficile en périphérie et l'administration mal codifiée [8]. La prise en charge défectueuse, par la carence des structures de santé ou l'absence de matériel et de médicament appropriés fréquent dans de nombreux pays en développement, augmente le risque d'évolution défavorable quel que soit le délai de consultation. Les premiers soins lorsqu'ils sont agressifs, garrot, incision et scarification, risquent de réduire la circulation sanguine, d'infecter la plaie ou provoquer des hémorragies [9]. La toxicité et la quantité du venin injecté par le serpent sont des éléments essentiels. Ces facteurs dépendent de l'espèce de serpent, de sa taille, de la capacité des glandes à venin, de leur état de réplétion et des circonstances de morsures. L'âge, le poids, l'état de santé de la victime, le siège de la morsure et le délai de prise en charge sont également des éléments importants [9]. La morbidité est estimée à 10,52\% et la mortalité à 7,89\%. [10]. En milieu rural, la létalité reste élevée (supérieur à 15\%) en raison de l'indisponibilité du sérum antivenimeux (SAV) dont le coût et les difficultés de conservation apparaissent comme dissuasifs [11]. En ville la létalité quoique plus basse (inférieure à $4 \%$ ), grâce à un protocole de sérothérapie demeure inacceptable à cause des délais d'évacuation vers l'hôpital national [12]. II ressort d'une enquête que seulement 0,68 \% des morsures sont adressés au centre de santé au profit de la tradithérapie. Au niveau local, la prise en charge des morsures est assurée au niveau familial ou par le guérisseur du village [13]. L'incidence des envenimations ophidiennes reste largement sousestimée dans les pays tropicaux.

Au Mali, L'incidence annuelle est de 164 morsures pour 100000 habitants et la totalité des informations actuelles accumulées sur les morsures de serpents relève du milieu urbain de Bamako [13]. Cependant en milieu rural, les morsures de serpents sont des accidents de travail agro-pastoral qui mériteraient plus d'attention en raison de leur fréquence et de leur sévérité. [13]. II n'y avait pas eu d'étude similaire dans notre site, un seul agent sur les huit personnels du centre est formé sur la prise en charge des cas de morsure du serpent, le centre s'approvisionne en SAV à travers un distributeur agréer de Bamako et vend un flacon à vingt et cinq mille F CFA ; d'où l'intérêt de notre étude d'évaluer la prise en charge des morsures de serpent en milieu rural.

\section{Matériel et méthodes :}

II s'agissait d'une étude transversale descriptive et prospective qui s'est déroulée de Janvier à Décembre 2020 dans l'aire du centre de santé communautaire universitaire (CSCom-U) de Ségué, dans le district sanitaire de Kolokani dans la région de Koulikoro. Le CSCom-U de Ségué est un établissement rural qui regroupe 8 villages et 6 hameaux avec une population de 5049 habitants selon le Recensement Générale de la Population et de l'Habitat (RGPH4). Les habitants sont en majorité des agriculteurs et des éleveurs.

La population d'étude était constituée des victimes de morsures de serpent vues en consultation au CSCom-U de Ségué et des victimes ou leurs parents dans les ménages de l'aire de santé avec l'appui des relais communautaires.

Les variables étudiées portaient sur les caractéristiques sociodémographiques du malade (âge, sexe, résidence, profession), le délai entre la morsure présumée et l'admission au CSCom- $U$, les circonstances de la morsure, le siège de la morsure, le tableau clinique, le traitement et l'évolution, le bilan biologique qui portait sur le taux d'hémoglobine.

Le tableau clinique permettait de classer les victimes d'envenimation en stades suivants [12]: Grade 0 : Pas d'envenimation, pas œdèmes, pas réactions locales.

Grade 1 : Forme bénigne (envenimation minime), les signes locaux sont discrets (traces de 2 crochets, avec enflure inflammatoire et douloureuse). Absence de manifestation.

Grade 2 : Envenimation modérée, les signes locaux sont présents, un œdème inflammatoire douloureux d'apparition rapide (toujours avant la 30 ème minute), une douleur vive au point d'injection du venin, les traces de crochets entourés d'une auréole rouge parfois invisible. Les signes généraux sont présents mais restent modérés.

Grade 3 : Envenimation sévère, sur le plan cardiorespiratoire : un état de choc avec anurie secondaire, un arrêt cardiaque exceptionnel, une détresse respiratoire aiguë avec œdème laryngé. Extension de l'œdème, elle se fait vers la région cervicale entraînant un œdème pharyngolaryngé mortel. Sur le plan hématologique, une coagulopathie de consommation.

Les données ont été recueillies à partir des questionnaires individuels pour les ménages et pour le centre.

La saisie des données a été faite sur le logiciel Access 2016. L'analyse des données a été faite à l'aide du logiciel SPSS 16.0. Les tests statistiques utilisés étaient le Khi2 et l'Exact de Fisher avec un seuil de significativité de 0,05. Le consentement libre et éclairé a été demandé et obtenu auprès de chaque participant, les principes de l'anonymat et de la confidentialité étaient de rigueur.

\section{Résultats :}

Nous avons enregistré 25 cas de morsure de serpent durant la période d'étude, dont 10 cas (40\%) au centre et $15(60 \%)$ dans la communauté. La tranche d'âge 15 à 30 ans était la plus représentée (40\%) et le sexe masculin était le plus touché soit $64 \%$ (Tableau I).

La saison des pluies a enregistré plus de cas de morsures avec $64 \%$ suivie des saisons froide $(24 \%)$ et sèche $(12 \%)$. 
La plupart des morsures (76\%) a eu lieu en brousse ( $p$ $=0,001$ ) et la circonstance la plus fréquente était les travaux champêtres (40\%). Les professions cultivateur et ménagère étaient les plus fréquentes avec respectivement $36 \%$ et $28 \%$ contre $8 \%$ d'élèves.

Le point de morsure a concerné le pied dans $72 \%$ des cas contre $28 \%$ pour la main. Le type de serpent a été identifié dans $80 \%$ des cas et était tous des vipères (100\%). Parmi les victimes prises en charges au centre, $70 \%$ avaient utilisé au moins un médicament traditionnel avant d'arriver. Seulement 4 sur 10 (40\%) étaient arrivés au centre dans un délai de 02 heures qui ont suivi la morsure. Les 14 sur les 15 prises en charge dans la communauté (93\%) étaient arrivés dans un délai de 02 heures chez un tradithérapeute. Le choix de prise en charge traditionnelle était motivé par le coût élevé du sérum anti venimeux $(69 \%)$ et la confiance aux médicaments traditionnel (31\%). L'utilisation de garrot $(24 \%)$, de pierre noire $(8 \%)$ et l'incision (32\%) étaient les premiers gestes pratiqués avant d'aller au lieu de prise en charge.

L'envenimation a été enregistrée chez 24 malades sur 25 soit $96 \%$, classée à des grades suivant : Grade1 $=20 \%$, Grade $2=68 \%$ et Grade $3=8 \%$. Les signes généraux étaient dominés par la fièvre et le vomissement dans $36 \%$ des cas pour chacun, les céphalées et vertiges dans $20 \%$ également (Figure 1). Les signes locaux dominant étaient par la présence de traces de crochets dans $100 \%$ des cas, les œdèmes dans $96 \%$ des cas et le saignement du site de morsure dans $84 \%$ des cas (Figure 2). Le syndrome hémorragique était présent dans $32 \%$ des cas soit $8 / 25$ dominé par le saignement local, la gingivorragie et l'épistaxis (Figure 3). Le niveau d'œdème, ne dépassant pas le coude ou le genou était $84 \%$ des cas contre $12 \%$ dépassant le coude ou le genou. Le syndrome vipérin a été retenu dans $100 \%$ des cas d'envenimation. Les taux d'hémoglobines étaient normaux chez tous les malades traités au centre. Tous les patients pris en charge au centre de santé ont reçu au moins une dose de Sérum Anti Venimeux (SAV) dont $60 \%$ ont reçu deux ampoules et $40 \%$ une ampoule. Le temps de rémission des signes cliniques était de moins de 24 heures chez tous les patients pris en charge au centre contre plus de 72 heures chez 12 sur 15 des patients pris en charge par les tradithérapeutes $(p=0,001)$ (Tableau II). Nous avons enregistré $100 \%$ de guérison dont un seul cas avec séquelle qui avait été traité traditionnellement. Le temps de rémission des signes était lié au grade $(p=0,038)$.

\section{Discussion :}

Les limites de cette étude ont surtout été l'absence de contact direct avec les guérisseurs traditionnels de morsure de serpent courant l'étude, afin de faire leur cartographie pour une éventuelle collaboration. Compte tenu de l'insuffisance du plateau technique, seul le taux d'hémoglobine était réalisable.

Les points forts ont été l'évaluation à temps réel la fréquence, le mode de prise en charge et l'évolution des cas de morsure de serpent dans la communauté. A cela s'ajoute la disponibilité et la bonne conservation des sérums antivenimeux au centre de santé.

\section{Sur le plan épidémiologique}

Nous avons enregistré, 25 cas de morsure de serpent durant la période d'étude. La morsure du serpent a représenté $0,36 \%$ des pathologies enregistrées dans le CSCom U en 2020 (10/2773). Dramé B. et al. et de Coulibaly S K et al. trouvent respectivement 112 et 156 cas de morsure. [12,14].

La tranche d'âge 15 à 30 ans et le sexe masculin étaient les plus fréquents. Chippaux J.P en 2002 trouve que les hommes subissent entre 50 et $75 \%$ des morsures. Les femmes et les enfants sont moins souvent mordus [5]. La saison des pluies a enregistré plus de cas de morsures. La reproduction des ophidiens en zone de savane pendant la saison des pluies expliquerait la recrudescence des morsures durant cette période [13].

Plus de trois quarts des morsures a eu lieu en brousse $(p=0,001)$ et la circonstance la plus fréquente était les travaux champêtres. Les professions cultivateur et ménagère étaient les plus fréquentes. Selon Chippaux J.P en 1992, les trois quarts des morsures surviennent au cours des travaux agricoles, de la chasse ou du déplacement pédestres en rapport avec le travail [4].

Le point de morsure a beaucoup concerné le pied. Les membres inferieurs sont les plus exposés en raison de contact fréquent avec le sol qui est l'habitat préféré des vipères.

Le type de serpent impliqué dans les morsures était les vipères. Les vipères sont les espèces de serpents venimeux les plus responsables de morsure dans le monde [3].

\section{Sur le plan Clinique}

Les signes étaient dominés par la fièvre, le vomissement, les céphalées, les vertiges, les œdèmes, le saignement du site de morsure, la gingivorragie et l'épistaxis. II n'y avait pas de signes neurologiques contrairement à l'étude de B Dramé et al. qui trouvent $10,7 \%$ de cas de signes neurologiques [12]. En tous, nos cas d'envenimations étaient de syndrome vipérin qui ne donne pas de manifestations neurologiques.

\section{Sur le plan thérapeutique et évolution}

Dans notre étude moins de la moitié a fait recours au centre de santé. Ce résultat confirme le faible recours aux soins médicaux trouvé par Dabo $A$ et al. qui recensent $0,68 \%$ cas adressé à une structure de santé [13]. Le coût de l'ordonnance et la croyance aux soins traditionnels sont autant de facteurs qui expliqueraient le faible taux de fréquentation des centres de santé. Par ailleurs, parmi les victimes prises en charge au centre, $70 \%$ avaient utilisé au moins un médicament traditionnel avant d'arriver. Cela dénote la confiance de la population au médicament traditionnel dans la prise en charge de la morsure de serpent. II serait intéressant de mener une étude plus approfondie les raisons qui motive ce recours à la médecine traditionnelle. Le délai de prise en charge dans les 02 heures qui ont suivi la morsure était plus fréquent chez les victimes qui ont eu recours aux guérisseurs qu'au 
centre de santé. Cela pourrait être expliqué par la proximité des guérisseurs aux victimes.

Les premiers gestes pratiqués avant le lieu de prise en charge étaient dominés par l'utilisation de garrot, de pierre noire et l'incision. Coulibaly S K et al. trouvent $31 \%$ de cas d'utilisation de pierre noire [14]. Ces deux résultats prouvent la méconnaissance de la population sur les bonnes pratiques (pas de garrot, pas d'incision, recours précoce aux soins de santé) face à la morsure de serpent. Le temps de rémission des signes cliniques était significativement plus rapide au centre que chez les tradithérapeutes $(p=0,001)$. Ce résultat pourrait être dû à la bonne qualité et à l'utilisation appropriée du SAV par les personnels. Nous avons enregistré $100 \%$ de guérison dont un seul cas de séquelle (atrophie musculaire du pied avec une légère boiterie) chez un patient traité traditionnellement, cette séquelle pourrait être due au retard d'un traitement efficace et approprié car le malade avait fait le tour chez plus de deux tradithérapeutes.

\section{Conclusion :}

La morsure de serpents est un évènement assez négligé dans nos programmes de santé, très souvent les victimes font recours aux soins traditionnels du fait des croyances culturelles mais aussi à l'accès difficile au sérum antivenimeux dont le coût n'est pas à leur porté, or l'utilisation du sérum antivenimeux est le traitement de choix. La disponibilité au niveau local des SAV, la formation continue des agents de santé, et l'éducation de la population doivent être de mise pour améliorer la prise en charge communautaire des envenimations par morsure de serpent.

\section{Références :}

1. CHIPPAUX J.P. : Serpent d'Afrique Occidentale et Centrale. Les Serpents et l'environnement. 2000.http://www.mpl.ird.fr/serpents/benin.htlm.

2. Rachida Soulaymani-Bencheikh : Morsure de serpent Centre Anti Poison du Maroc N² 24 - 1er trimestre 2015. http://www.capm.ma

3. Gentilini M :(2003), Animaux venimeux. Flammarion Médecine Science, Cinquième édition, Paris, 1993 ; 715721.682pages.

4. Chippaux J P. les morsures de serpents en Afrique intertropicale. Cahier de santé, 1992, 2,221-234.

5. Chippaux J P : venin de serpent et envenimation. IRD, édition, Paris, 2002-288p.

6. Chippaux J P : 1998. Snake bite: appraisal of global situation. Bull.W.H.O, 76:515-524.

7. Bellefleur J P, LE Dantec $P$. Prise en charge hospitalière des morsures de serpents en Afrique.2005; Bull.soc.Pathol. Exot ; 98 ; 4 :273-276.
8. Chippaux J $P$, the development and use of immunotherapy in Africa. Toxicon, 1998, 76, 515-524.

9. Chippaux J P. Evaluation de la situation épidémiologique et des capacités de prise en charge des envenimations ophidiennes en Afrique subsaharienne francophone. Bull Soc Pathol Exot, 2005, 98, 4, 263-268 10. Toure MK, Coulibaly M, Kone J, Diarra MS, Coulibaly BB, Beye SA et al. Complications aigues de l'envenimation par morsures de serpent au service de réanimation du chu mère enfant "LE Luxembourg" de Bamako Mali MEDICAL 2019 TOME XXXIV N¹

11. Dabo A. Diawara SI, Dicko A, Katilé A, Diallo A, Doumbo 0 . Evaluation des morsures de serpents et leur traitement dans le village de bancoumana ; Au Mali. Bull Soc. Pathol. Exot, 2002, 95,184-187.

12. B Dramé, N Diani, M M Togo, M Maïga, D Diallo, A Traoré. Les accidents d'envenimations par morsure de serpent au service des urgences chirurgicales de l'hôpital Gabriel Touré, Bamako, Mali (1998-1999). Bull Soc Pathol Exot, 2005, 98, 4, 287-289.

13. Dabo A, Kouriba B, Traoré A, Diarra Y, Doumbo O. Morsures de serpents en zone soudano-sahélienne du Mali : Epidémiologie, symptomatologie et traitement. Med Trop 2010; 70: 49-52.

14. Coulibaly $S \mathrm{~K}$, Hami $H$, Mokhtari A, Soulaymani R, Maiga A, Soulaymani A, 2012. Problématiques des morsures de serpents dans la région de Mopti, Mali. Antropo, 28, 81-85. www.didac.ehu.es/antropo.

\section{LISTE DES TABLEAUX ET FIGURES}

Tableau I : Distribution des victimes en fonction de l'âge et du sexe.

\begin{tabular}{|c|c|c|c|}
\hline \multirow[b]{2}{*}{ Tranche d'âge } & \multicolumn{2}{|c|}{ Sexe } & \multirow[b]{2}{*}{ Total } \\
\hline & Féminin & Masculin & \\
\hline Moins de 14 ans & 2 & 6 & 8 \\
\hline Entre 15 et 30 ans & 2 & 8 & 10 \\
\hline 31 ans et plus & 5 & 2 & 7 \\
\hline Total & 9 & 16 & 25 \\
\hline
\end{tabular}

Tableau II : Temps de rémission des signes par rapport au type de traitement

\begin{tabular}{|c|c|c|c|}
\hline \multirow{2}{*}{$\begin{array}{l}\text { Temps de rémission } \\
\text { des signes }\end{array}$} & \multicolumn{2}{|c|}{ Type de traitement } & \multirow[b]{2}{*}{ Total } \\
\hline & Médical & Traditionnel & \\
\hline 24 heures & 10 & 0 & 10 \\
\hline $24 \mathrm{H}-48 \mathrm{H}$ & 0 & 2 & 2 \\
\hline $72 \mathrm{H}$ et plus & 0 & 12 & 12 \\
\hline Total & 10 & 14 & 24 \\
\hline
\end{tabular}




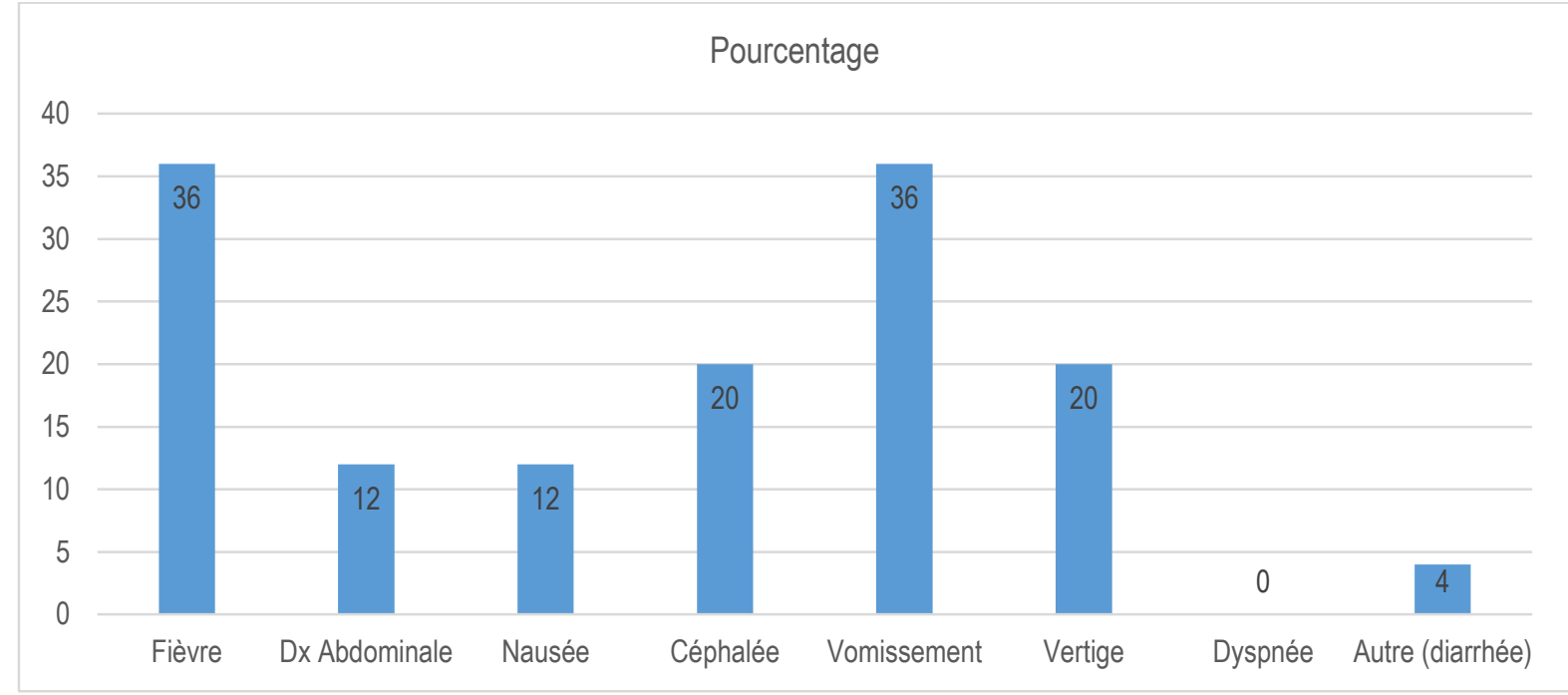

Figure 1 : Signes généraux

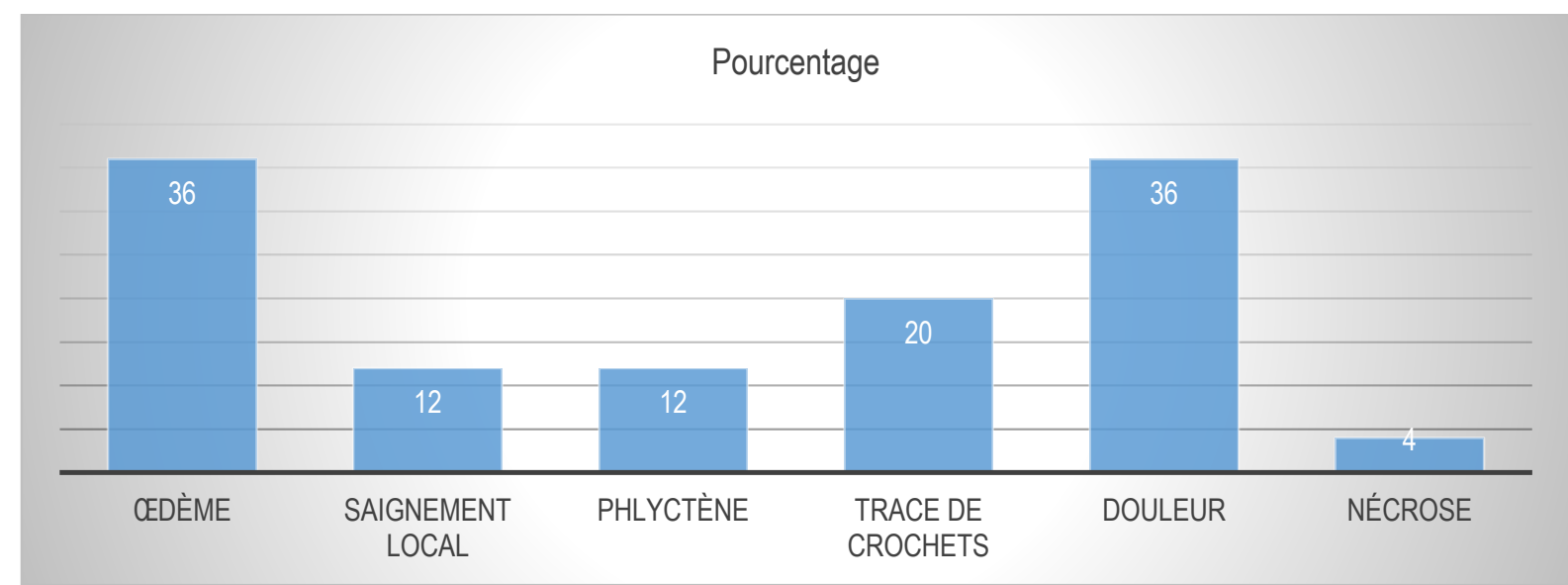

Figure 2 : Signes locaux

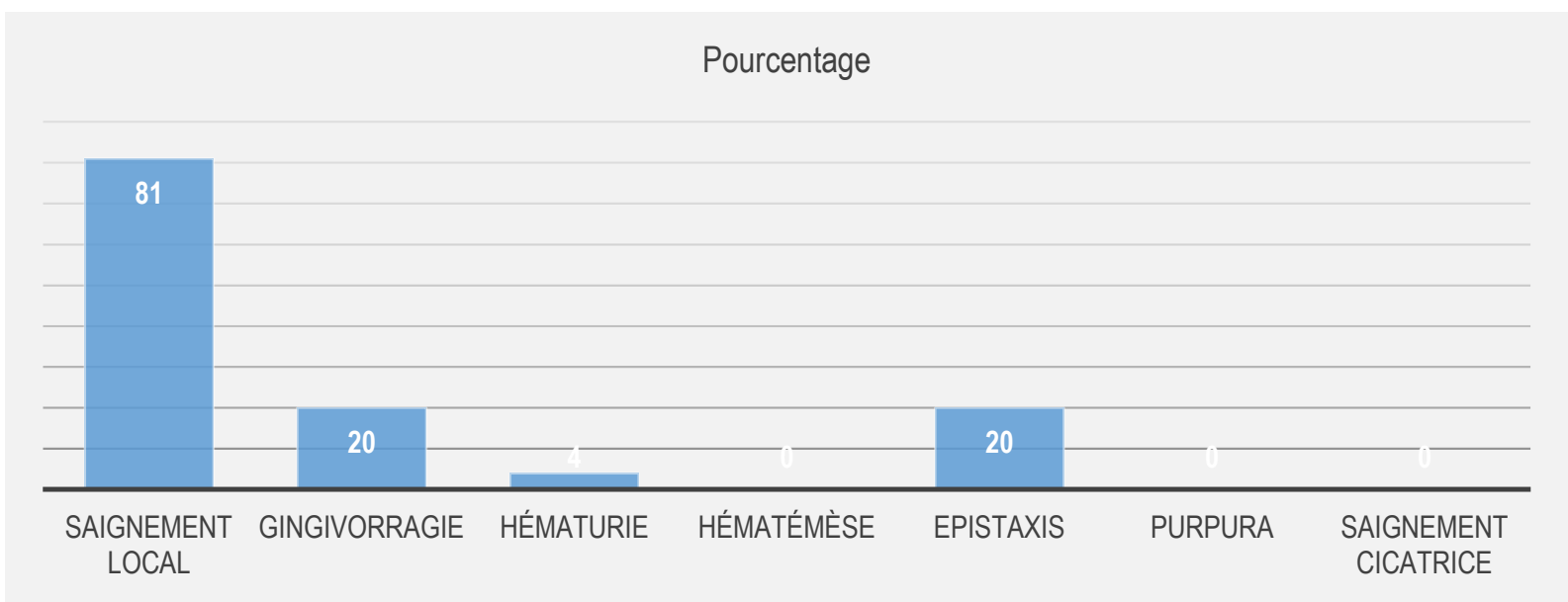

Figure 3 : Syndrome hémorragique 